\title{
Reciprocal predicates: a prototype model
}

\author{
Imke Kruitwagen, Yoad Winter \& James Hampton*
}

\begin{abstract}
Many languages have verbal stems like hug and marry whose intransitive realization is interpreted as reciprocal. Previous semantic analyses of such reciprocal intransitives rely on the assumption of symmetric participation. Thus, 'Sam and Julia hugged' is assumed to entail both 'Sam hugged Julia' and 'Julia hugged Sam'. In this paper we report experimental results that go against this assumption. It is shown that although symmetric participation is likely to be preferred by speakers, it is not a necessary condition for accepting sentences with reciprocal verbs. To analyze the reciprocal alternation, we propose that symmetric participation is a typical feature connecting the meanings of reciprocal and binary forms. This accounts for the optionality as well as to the preference of this feature. Further, our results show that agent intentionality often boosts the acceptability of sentences with reciprocal verbs. Accordingly, we propose that intentionality is another typical semantic feature of such verbs, separate from symmetric participation.
\end{abstract}

Keywords. Reciprocal predicates; verb meaning; prototype theory; typicality; experimental semantics; lexical semantics.

1. Introduction. Reciprocal verbs alternate between a unary and a binary form:

(1) Julia and Sam hugged.

(2) Sam hugged Julia.

In the intransitive sentence (1) the verb hug takes a plural agent, whereas (2) distinguishes two thematic roles. Sentences like (1) and (2) have a clear semantic relation with each other. Intuitively, sentence (1) reports a collective activity where the participants perform acts of the sort reported by (2). However, the specifications of this semantic relation are not easy to pinpoint. Most previous works have analyzed the semantics of alternations as in (1)-(2) under the assumption that reciprocal events as reported by (1) are obligatorily symmetric. Thus, these works rely on the introspective judgement that (1) logically entails (2). In contrast to these truth-conditional approaches, we propose that sentences like (1) support symmetric events as part of their most typical interpretation. Thus, (2) is true in the most typical events reported by (1) but it is not logically entailed by (1). This typicality approach is supported by experimental results on filmed scenarios where speakers accept reciprocal sentences (1) but reject sentences like (2). We propose that the typicality approach embodies a more adequate analysis of reciprocal verbs, which is also relevant for other alternations such as active-passive alternations or active-causative alternations.

\footnotetext{
* For their dedicated work on the materials for the experiment, we are thankful to Hilbert Dijkstra, Eva Kröse, Anna Peeters and Lianne Zandstra. This work was funded by the European Research Council (ERC) under the European Union's Horizon 2020 research and innovation programme (grant agreement No 742204).

Authors: Imke Kruitwagen, Utrecht University (i.kruitwagen@uu.nl), Yoad Winter, Utrecht University (y.winter@uu.nl) \& James Hampton, City, University London (j.a.hampton@city.ac.uk).
} 
Previous works followed different theoretical lines to analyze the reciprocal alternation. Gleitman (1965) and Lakoff \& Peters (1966) use transformational rules that connect the different verbal forms in a reciprocal alternation. Dowty (1991) analyzes the alternation by means of proto-roles, where the reciprocal form contains multiple proto-roles for agents, who act on each other according to the proto-roles for the binary form. Carlson (1998) on the other hand argues that the unary form comes with only one agent role, so different entities can carry the agent role together. Dimitriadis (2008) and Siloni (2008) strengthen Carlson's account, claiming that unary reciprocals necessarily denote an irreducible symmetric event. Gleitman et al. (1996) focus on the relation between reciprocity and symmetry of binary predicates. They point out that many predicates like hug, kiss, and fight do not show symmetry in their binary form. However, like the other aforementioned works, Gleitman et al. also assume that reciprocal entries uniformly require symmetric participation of the agents in the sense that is exemplified above for (1). Thus, while there are many technical and theoretical differences between the different approaches to lexical reciprocity, they all share the prediction about a logical entailment between sentences like (1) and (2): any speaker who accepts (1) as true is also expected to accept (2) as true. In short:

(3) Julia and Sam hugged $\rightarrow$ Sam hugged Julia and Julia hugged Sam.

We refer to this assumption on reciprocal verbs like hug as symmetric participation.

Intuitively, it is not clear that all reciprocal verbs indeed show symmetric participation in this way. Consider sentence (4) below, in a situation where a car collided with a stationary truck:

(4) The car and the truck collided.

Postulating symmetric participation for (4) would expect it to be judged false in this situation. However, when we informally consulted speakers about (4), the majority of them said they would accept (4) in the given scenario. To account for such judgements, our proposal challenges the assumption that sentences with unary reciprocals logically entail symmetric participation of subject entities. Instead of treating symmetric participation as necessary for the truth of sentences with reciprocal verbs, we hypothesize that it is only a preferred condition for the acceptance of such sentences. Thus, in typical situations where reciprocal sentences like (1) and (4) are considered true, the two corresponding binary sentences will be judged true as well, but there might be atypical situations where this judgement will not go through.

Most natural concepts have more than one typical attribute (e.g. a fruit is typically both round and sweet). We show that something similar holds of reciprocal verb concepts by identifying an attribute separate from symmetric participation that contributes to the typicality of reciprocal events. This attribute, which we call collective intentionality, describes the sum of relevant intentions and emotions of group members towards the event. In Sam and Julia hugged, we might expect Sam and Julia to be happy and affectionate. In Sam and Julia fought, Sam and Julia are likely to be angry and aggressive. Both symmetric participation and collective intentionality are assumed to contribute to the typicality of reciprocal events. Accordingly, we expect asymmetric participation to be more easily tolerated in case where the agents show the relevant kind of collective intentionality. For instance, we hypothesize that the possibility of judging (2) as false when (1) is true is boosted in situations where both Sam and Julia show affection to each other. In binary sentences where the agent is singular, we expect that only the agent's intentionality affects the 
acceptability of the sentence, whereas the intentionality of the other participant (patient or theme) is assumed to have a smaller effect.

The paper is structured as follows. Section 2 reports an experimental study of two hypotheses: (i) symmetric participation is not a necessary condition for reciprocal verbs; and (ii) collective agent intentionality can boost the acceptability of sentences with reciprocal verbs, whereas patient/theme intentionality does not have an equal effect on the acceptance of binary sentences. Section 3 discusses the results and proposes an account in terms of Hampton's (2007) threshold model.

2. Experiment. In the reported experiment, we collected truth-value judgements on sentences with unary and binary forms. Dutch speakers were shown situations where symmetric participation is likely to be missing, and which varied with respect to collective intentionality. Each experimental item consisted of a short video clip and a Dutch sentence, either a unary sentence of the form $A$ and $B$ verb (e.g. "Violet and Mark fought") or a binary sentence of the form $B$ verb (preposition) $A$ (e.g. "Mark fought (with) Violet"). All videos depict situations with two characters, one of whom visibly performs the relevant physical action on the other character, while the other character remains passive. For instance, for the verb knuffelen ("hug"), we used video clips that showed a woman ("Violet") clasping a man in her arms, while the man ("Mark") does not clasp her in his arms. Binary sentences (e.g. "Mark hugged Violet") all contained a subject that refers to the passive character, and are therefore expected to be judged as false. If sentences with unary forms are accepted despite rejection of the corresponding binary sentences, we conclude that the unary form does not require symmetric participation.

The video clips that were used were of two types. In one type, both characters showed the intention that is intuitively expected with respect to the physical act (e.g. affection for hug, anger for fight). We consider these video clips as illustrating collective intentionality. In the other type of video, only the active character showed the expected intention, whereas the reaction of the passive character was unsuitable to the act (e.g. disgust for hug, indifference for fight). Both types of video were designed as lacking the relevant physical act of the "passive" character. Thus, if sentences with the unary form are accepted significantly more in the situations with collective intentionality despite equal acceptance of the binary sentence, we conclude that collective intentionality boosts the acceptance of sentences with unary reciprocal forms.

\subsection{METHOD.}

2.1.1. PARTICIPANTS. A total of 449 participants (287 female, age $M=19$ ) took part in the experiment. They were all enrolled in a first year bachelor's course at Utrecht University and they took part in the experiment as part of a class. Participants did not receive monetary compensation.

2.1.2. MATERIALS. The unary and binary forms of four Dutch reciprocal verbs were tested for their acceptability, each of them in two different situations: with/without collective intentionality. In total there were 16 test items, each of which consisting of a short video clip and a Dutch sentence. In addition, participants were asked about their age, gender and native language.

Target item sentences were constructed from verbs that have both a binary form (e.g. "A hugged B") and a unary form with a collective interpretation (e.g. "A and B hugged"). In order to optimally represent the semantic variety of reciprocal verbs, these four verbs were selected:
- knuffelen
(hug)
- botsen (tegen)
(collide (with))
- vechten (tegen)
(fight (against)) 
- fluisteren (tegen) (whisper (to))

Unary and binary target sentences were included for each verb. In addition to the 8 target item sentences, 8 filler item sentences were constructed. The filler items served to obtain a better balance in the expected number of true/false reactions and to prevent the participants from using automatic strategies when reacting to the target stimuli. The filler item sentences included both unary and binary verbs.

For each target verb, two different videos were used. The videos were approximately 30 seconds long, showing a woman ("Violet") and a man ("Mark"). In all target videos, the woman carries out the action described by the verb. For instance, in case of hug, she wraps her arms around the man; in case of talk, she speaks. The man remains passive and does not physically carry out the physical actions described by the verb. In case of hug, for instance, the man does not wrap his arms around the woman; in case of talk, he does not speak. The difference between the two videos for each verb is in the intentionality of the man. One type of videos, henceforth "CI videos", demonstrated collective intentionality in that the man uses social cues such as facial expressions and body language to show his involvement in the action. The second type of videos, "no-CI videos", is similar to the CI videos except for one respect: the man now expresses a negative attitude towards the action or is indifferent towards it.

We used a between-subject design with a total of eight versions. Each version contained three items: a target item with a unary sentence, a target item with a binary sentence, and a filler item. The verbs in the three items were all different. Four versions contained only target items with CI videos, while the four other versions only contained target items with no-CI videos. This was done to prevent participants from consciously noticing a difference in the man's involvement towards the action, which could influence their judgements.

2.1.3. ProcedurE. The experiment was designed using LimeSurvey and an application developed at Utrecht University with the aim of randomizing the allocation of participants to questionnaires (https://rocky.sites.uu.nl/tools/). The experiment was run in a lecture room during the break of a lecture. The url to the experiment was presented on a large screen. Following the url led participants to a randomized version of the experiment. Participants used earphones and individual laptop, tablet or smart phone. For each item, a video was displayed, after which the accompanying sentence was shown on the screen. Participants were asked to indicate whether they judged the sentence as "true" or "false" in the preceding video. They were told that there are no right or wrong answers, but that we as researchers were curious about their intuition. They were also instructed to not think too long about their judgement.

2.1.4. ANALYSIS. Fifteen participants were excluded from analysis because they were not native speakers of Dutch. For each of the target items, the proportion of "true"-responses was computed. Then, a logistic regression model was constructed, using Sentence Type (Unary or Binary) and video Intention (CI or no-CI) as predictor variables.

2.2. RESUlTS. The results demonstrate a difference in acceptance rates between all four target types, as shown on Figure 1. 


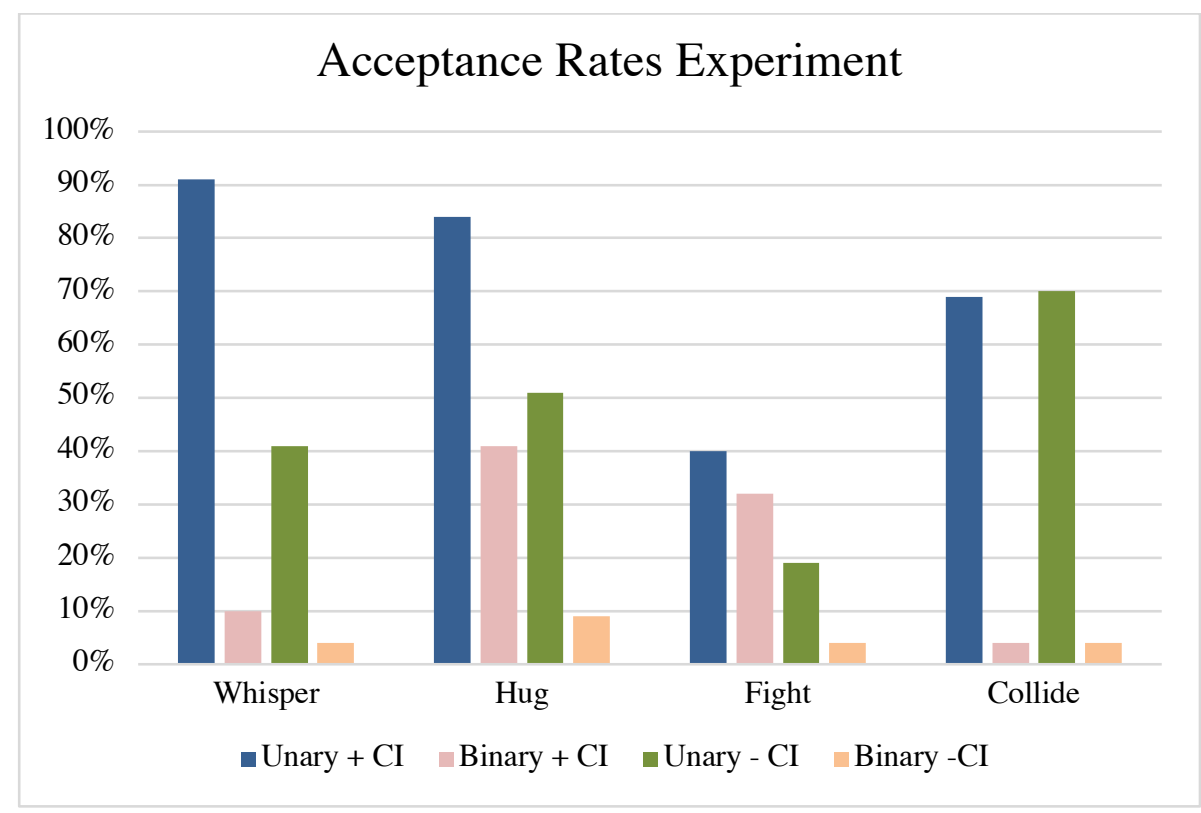

Figure 1: Results Experiment

With respect to the CI videos, there is a substantial difference between acceptance rates on the unary and binary forms. For unary forms with a CI video the acceptance rates varied from $40 \%$ for fight to $90 \%$ for whisper, while the acceptance rates for binary forms on CI videos varied from $4 \%$ for collide to $39 \%$ for hug. Furthermore, there is also a substantial difference between acceptance rates on unary forms in CI videos and unary forms in no-CI videos. Acceptance rates for unary sentences in no-CI videos varied from $18 \%$ for fight to $69 \%$ for collide. However, for collide there was no difference between the acceptance rates on the unary sentence with a CI video and the unary sentence with a no-CI video.

Logistic regression analyses were computed, using Sentence Type (Unary vs. Binary), Intention $(\mathrm{CI}$ vs. no-CI) and Sentence Type $\times$ Intention interaction as predictor variables. Separate analyses were done for each of the four verbs. For all verbs a significant effect of Sentence Type was found. Thus, sentences with the unary form of the verb yielded significantly more true answers when compared to sentences with the binary form. For all verbs except collide there was a significant effect of Intention on the acceptance of both unary and binary form sentences. Thus, except for collide, unary and binary form sentences combined with the CI videos were accepted more frequently than unary and binary form sentences combined with no-CI videos.

No verb exhibited a significant interaction effect between Intention and Sentence Type. Thus, there was no statistical evidence that Intention had a larger effect on acceptance of unary forms than on binary forms.

3. Discussion. Previous proposals on the semantics of reciprocal alternations assume symmetric participation: a logical entailment from sentences with the unary form ("Violet and Mark hugged") to their binary counterparts ("Violet hugged Mark" and "Mark hugged Violet"). While this entailment is very likely to hold for reciprocal verbs like marry and date, our work questions it for verbs like hug or collide. As an alternative, the following hypotheses were tested: (i) symmetric participation is not a necessary condition for reciprocal verbs; and (ii) collective intentionality can boost the acceptability of sentences with unary form reciprocals, beyond its possible effect on the acceptance of binary form sentences. Our results showed situations where unary sentences yield 
significantly more true answers compared to their binary correlates. Thus, while many participants saw that the event lacked symmetric participation, this did not prevent many participants from judging the unary sentences as true. It is unlikely that symmetric participation holds for all speakers. This supports hypothesis (i) and is evidence against the entailment theory.

As for our second hypothesis, with 3 of the 4 verbs that were tested (hug, fight and whisper), collective intentionality significantly boosted the acceptance of both unary and binary sentences. There was no statistical evidence that collective intentionality had a larger effect on the acceptance of unary form reciprocals than on binary forms. Hypothesis (ii) is thus only partially supported. For collide there was no effect of collective intentionality at all. This is not surprising: typically, there is no intentionality involved in an event of colliding. For the other verbs, it is tempting to conclude that collective intentionality is a typical feature for both the unary and the binary from of reciprocal verbs. Thus, surprisingly, the intentionality of a non-agent participant might matter as much as that of the agent's. If this is the case, effects of collective intentionality on acceptance of reciprocal verbs may only be an epiphenomenon of its effect on binary forms: the acceptability of unary form sentences may depend only on the acceptability of their binary correlates. Under this interpretation, since collective intentionality positively influences acceptance of the binary form, it indirectly affects acceptance of the unary form as well. This line of explanation is in line with Gleitman et al.'s (1996) proposal that binary forms of (at least some) reciprocal verbs have typical symmetry about them, even if the symmetric participation that Gleitman et al. assume for reciprocal entries is not supported by our results.

Our experimental results lead to a new proposal regarding the reciprocal alternation. Instead of the logical entailment that is involved in the idea of symmetric participation, we propose a typicality approach, where conceptual features connect the unary and binary form. The conceptual features that are shared by all reciprocal verbs are symmetric participation and collective intentionality. The combination of the verb form (unary or binary) and the conceptual content of its stem determines the typicality of different situations for sentences made of the verb. If one conceptual feature has a non-typical value (in our case, lack of symmetric participation), the other conceptual feature (in our case, collective intentionality) can boost the overall typicality of the situation. In such cases the event may end up passing the threshold of belonging to the relevant category of events despite the lack of one of the typical features as in Hampton's (2007) threshold model. Consequently, the corresponding sentence may become acceptable.

Different verb concepts may have different weights assigned to relevant features. For example, for collide, collective intentionality is not an important conceptual feature: the contribution of collective intentionality to event typicality is low, or even zero. By contrast, for whisper, collective intentionality is proposed to be an important feature, which explains why the acceptance of "Violet and Mark whispered" increases significantly when Violet and Mark show collective intentionality, compared to a minimally different event in which Mark does not show intention. A remaining question that our study leaves open is to what extent collective intentionality is specifically a typical property of unary forms, and to what extent it also characterizes binary forms that participate in reciprocal alternations.

\section{References}

Carlson, Greg. 1998. Thematic roles and the individuation of events. Events and Grammar, 3551. Springer. https://doi.org/10.1007/978-94-011-3969-4_3 
Dimitriadis, Alexis. 2008. Irreducible symmetry in reciprocal constructions. In Ekkehard König \& Volker Gast (eds), Reciprocals and reflexives: Theoretical and typological explorations. Trends in linguistics 192, 375-409. Berlin/New York: Mouton de Gruyter. https://doi.org/10.1515/9783110199147.375.

Dowty, David. 1991. Thematic proto-roles and argument selection. Language, 547-619. https://doi.org/10.2307/415037.

Gleitman, Lila R. 1965. Coordinating conjunctions in English. Language, 41(2), 260-293. https://doi.org/10.2307/411878.

Gleitman, Lila. R., Harry Gleitman, Carol Miller \& Ruth Ostrin. 1996. Similar, and similar concepts. Cognition, 58(3), 321-376. https://doi.org/10.1016/0010-0277(95)00686-9.

Hampton, James. 2007. Typicality, graded membership, and vagueness. Cognitive Science, 31(3), 355-384. https://doi.org/10.1080/15326900701326402.

Lakoff, George, and Stanley Peters. 1966. Phrasal Conjunction and Symmetric Predicates. Report NSF-17, Harvard Computation Lab. Reprinted in Reibel and Schane ed., Modern Studies in English, Prentice Hall. 1969.

Siloni, Tal. 2008. The syntax of reciprocal verbs: An overview. In Ekkehard König \& Volker Gast (eds), Reciprocals and reflexives: Theoretical and typological explorations. Trends in linguistics 192, 451-498. Berlin/New York: Mouton de Gruyter. 451498. https://doi.org/10.1515/9783110199147.451. 